\title{
Experimental Study on the Calibration of Microparameters of Dolomite in the Barun Open-Pit Mine on the Basis of the Parallel Bond Model
}

\author{
Zhiheng Dang, ${ }^{1}$ Zuoming Yin $\mathbb{D}^{1},{ }^{1}$ Desheng Wang $\mathbb{D}^{1},{ }^{1}$ Mingyu Fu, ${ }^{2}$ and Qi Yin ${ }^{3}$ \\ ${ }^{1}$ Shool of Civil \& Resource Engineering, University of Science and Technology Beijing, Beijing, 100083, China \\ ${ }^{2}$ Inner Mongolia Baotou Steel Union Co., LTD, Barun Mining Branch, Baotou 014000, China \\ ${ }^{3}$ School of Management Engineering, Qingdao University of Technology, Qingdao 266033, China
}

Correspondence should be addressed to Zuoming Yin; yinzuoming@xs.ustb.edu.cn

Received 10 April 2021; Revised 14 July 2021; Accepted 2 December 2021; Published 21 December 2021

Academic Editor: Massimo Latour

Copyright $(2021$ Zhiheng Dang et al. This is an open access article distributed under the Creative Commons Attribution License, which permits unrestricted use, distribution, and reproduction in any medium, provided the original work is properly cited.

The microparameter calibration of the particle flow parallel bond model (PBM) is mostly based on a uniaxial compression test. The microparameters calibrated only by uniaxial compression tests cannot be directly used to study the mechanical properties of rocks with surrounding pressure conditions. To analyze the relationship between the macroparameters and microparameters in the model and select appropriate particle flow model parameters, this study conducted a particle flow numerical simulation experiment based on the basic test principles of the uniaxial compression, Brazilian splitting, and triaxial compression tests. An orthogonal experimental design was performed for the calibration of the microparameters of the particle flow PBM, and multifactor analysis of variance was used to screen out the factors that have a considerable influence on the experimental indicators. Regression analysis was performed on the significant influencing factors and test indicators, and the corresponding linear and nonlinear relationships between the macroparameters and microparameters were obtained. Lastly, the microparameters of the model were determined in accordance with the macroparameters of the mechanical test of the Barun open-pit mine dolomite, and a numerical simulation test was conducted. Simulation test results were consistent with the actual test results, thus providing a basis for a subsequent numerical simulation study on the mechanical properties of dolomite.

\section{Introduction}

The basic unit of the particle flow code (PFC) is composed of particles and contacts. It is a simplification of the discrete element method, which solves the problem of complex deformation models through a discontinuous method [1]. The finite difference and finite element methods are based on continuum mechanics, and it is difficult to simulate the whole process of rock crack generation, expansion, and failure. Nevertheless, the particle discrete element can automatically simulate the generation, expansion, and penetration of random cracks. It is currently widely used in coal mines, geotechnical engineering, and other related fields [2].

The constitutive model of particle flow simulation needs to

determine the microparameters that match the macroparameters of the material. Then, the microparameters are constantly adjusted to match the macromechanical properties. Most studies generally adopt the "trial-and-error method" for calibration, which is a blind and time-consuming adjustment process. Calibration methods have been studied by domestic and foreign scholars $[3,4]$, and the relationship between the macroparameters and microparameters of the particles has been explored. On the basis of the equivalent mesobeam of the contact bond model, Liu et al. [5] determined the relationship between the microscopic strength parameters and the fracture toughness of rocks. The particle contact interface of contact bond models cannot resist the torque; thus, the parallel bond model (PBM) is widely used to simulate rock materials. By combining theoretical analysis with numerical simulation, 
Cong et al. [6] systematically analyzed the influence of the microparameters of the PBM on its macroparameters and provided a quantitative relationship between them. Potyondy and Cundall [7] systematically summarized the application of the PBM in the simulation test of the Lac du Bonnet granite tunneling project and provided a method to calibrate the microparameters of the model. Xu et al. [8] investigated a method of selecting microparameters in the PBM and simulated the mechanical properties of limestone. Nardin and Schrefler [9] used a built-in model to simulate uniaxial compression and tensile tests of bonded materials and analyzed the interconnection between the macroparameters and microparameters by the dimensional method. Yang et al. [10] used the PBM to simulate twodimensional uniaxial compression tests on bonded particle materials; this approach could simulate the compression effect well. Huang [11] investigated the relationship between the macroparameters and microparameters in the built-in contact bond model of PFC2D and found that compressive strength is mainly influenced by cohesive strength and friction coefficient. Zhao et al. [12] combined theoretical analysis with numerical simulation and obtained quantitative relationships between the macroparameters and microparameters of the PBM. Yan et al. [13] designed orthogonal experiments for unbonded particle materials and studied the relationship between the macroparameters and microparameters. To calibrate the friction coefficient between sand and soil particles, Chen et al. [14] conducted three-dimensional particle flow simulation tests and studied the correlation between friction angle and earth pressure at rest. Zhou et al. [15] used a BP neural network method to establish a nonlinear model of macroparameters and microparameters and inputted macroparameters to invert the microparameters of the soil body. Niu and Xin [16] used multifactor analysis of variance to research the effects between the macroparameters and microparameters and matched microparameters with uniaxial compression tests. The above studies showed that, by choosing suitable model microparameters, particle discrete elements can simulate uniaxial (triaxial) compression tests and Brazilian splitting tests of rocks, and they are consistent with the results of the actual test. However, Chen and Hao et al. $[17,18]$ showed that the microparameters calibrated only by uniaxial compression tests cannot be directly used to study the mechanical properties of rocks with surrounding pressure conditions. Most rocks in nature are in a three-way stress state. Studying the strength and deformation characteristics of rocks in a three-way stress state is important to guide geotechnical engineering design. Thus, this study selects the PBM as the contact model and uses uniaxial compression, Brazilian split, and triaxial compression tests as the macroparameter testing methods [19]. An orthogonal table design numerical simulation test scheme is adopted, and multifactor analysis of variance is combined to study the influence of microparameters on its macroparameters, thereby providing basis for matching the microparameters of the particle flow model.

\section{Test Principle}

\subsection{Static Mechanical Test of Dolomite}

2.1.1. Rock Density. For rocks that can be prepared into regular samples, the most commonly used indoor measurement method is the volumetric method. When measuring rock density by the volumetric method, the average cross-sectional area $A$, average height $h$, and sample weight $m$ must be determined. The density of the rock is calculated by the following formula:

$$
\rho=\frac{m}{A \cdot h} .
$$

2.1.2. Uniaxial Compression Test. A cylindrical specimen is placed in the center of the pressure-bearing plate of the press and pressurized at a loading rate of 0.2 0.8 MPa per second. The sample is broken, and the load is stopped, and the uniaxial compressive strength UCS is obtained. According to the monitor of the deformation of the specimen, the elastic modulus $E$ and Poisson ratio $v$ of the rock are obtained. The calculation formulas are as follows:

$$
\begin{aligned}
& E=\frac{\sigma_{c(50)}}{\varepsilon_{h(50)}}, \\
& \mu=\frac{\varepsilon_{d(50)}}{\varepsilon_{h(50)}},
\end{aligned}
$$

where $\sigma_{c(50)}$ is $50 \%$ of the uniaxial compressive strength and $\varepsilon_{h(50)}$ and $\varepsilon_{d(50)}$ are the corresponding axial compressive strain and radial tensile strain at $\sigma_{c(50)}$. The final failure form of the specimen is shown in Figure 1.

2.1.3. Brazilian Split Test. The cushion strip changes the applied pressure into a linear load and makes the test piece produce a tensile force perpendicular to the direction of the upper and lower loads. The test piece is damaged in tension along the radial direction (Figure 2). The formula for calculating the tensile strength of Brazilian split test is

$$
T S=\frac{2 P_{\max }}{(\pi D H)},
$$

where TS is the tensile strength, $P_{\max }$ is the failure load of specimen, and $D$ and $H$ are the diameter and height of the specimen.

2.1.4. Triaxial Compression Test. According to the axial stress $\sigma_{1}$ and the lateral stress $\sigma_{3}$, the ultimate principle stress relationship curve is fitted, and the internal friction angle $\theta$ and cohesive force $C$ of the rock are obtained. The calculation processes of the internal friction angle $\theta$ and cohesive force $C$ are as follows: 


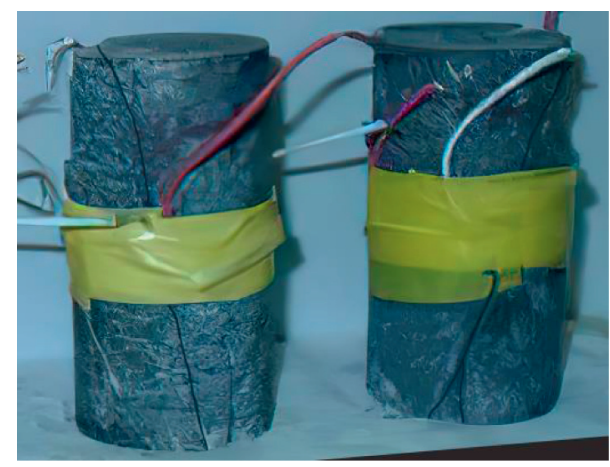

Figure 1: Rock failure under the uniaxial compression test.

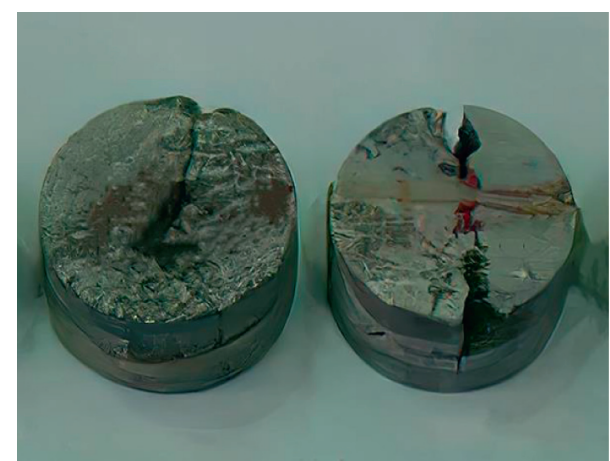

Figure 2: Rock failure under the Brazilian split test.

$$
\begin{gathered}
\theta=\arcsin \frac{k-1}{k+1}, \\
C=\frac{\sigma_{c}(1-\sin \theta)}{2 \cos \theta},
\end{gathered}
$$

where $k$ is the slope of the ultimate principle stress $\sigma_{1} \sim \sigma_{3}$ relationship curve and $\sigma_{c}$ is the intercept of the ultimate principle stress relationship curve on the ordinate axis.

The basic mechanical properties of dolomite are shown in Table 1.

\subsection{Particle Flow Parallel Bond Contact Model. Two basic} bond models, namely, the CBM and the PBM, are used in the particle flow model. The contact bond only occurs in a small area at the contact point, whereas the parallel bond occurs in the rectangular or circular cross section between the particles. The PBM can transmit not only force but also torque. A PBM is a bonding material model with constant rigidity (normal and tangential) and a certain strength between two particles. The parallel bond strength characteristic conforms to the Mohr-Coulomb criterion. When it fails, the PBM changes from bonded to unbonded state. The PBM in the unbonded state is equivalent to the linear contact model. The contact plane of the PBM has a certain size, which can transmit force and moment. The relative displacement of the particle contact position generates force and moment in the bonded state, resulting in tangential and normal stress. Once the tangential and normal stresses exceed the bond strength of the parallel bond, the parallel bond breaks down $[7,20]$.
The PBM is suitable for simulating the failure of rock materials. The particle flow model composed of bonds between particles is affected by particle density, size distribution, sample shape, packing form, and bonding properties. The particle size of the model-generated particles is evenly distributed between the maximum particle size $\left(R_{\max }\right)$ and the minimum particle size $\left(R_{\min }\right)$. The microparameters of the PFC2D model of rock established by the PBM include two aspects, particle and bond microparameters, as shown in Table 2.

\section{Research on the Influence of Macroparameters and Microparameters and Calibration of Microparameters}

3.1. Model Establishment. The rock specimens for uniaxial and triaxial compression tests are rectangular specimens with a length $(L)$ of $80 \mathrm{~mm}$ and a width $(W)$ of $40 \mathrm{~mm}$. The rock sample size of the Brazilian split test is set to a circular sample with a diameter $(D)$ of $40 \mathrm{~mm}$. Abi et al. [21] studied the effect of particle size ratio on the macroscopic characteristics by changing the ratio of the maximum radius $R \max$ of the particle to the short side $W$ of the model. The study shows that when $W / R_{\max }>80$, each strength tends to a stable value, and the macroparameters are independent of porosity. In addition, considering the balance of calculation time and efficiency, $W / R_{\max }$ is appropriate to be $80 \sim 120$. The research of Yin et al. [22] showed that the internal scale ratio of the material is very small and has almost no effect on the model characteristics. However, it causes the calculation time to increase sharply. To ensure calculation accuracy and efficiency, the value range of $L / R_{\min }$ is set to $30 \sim 120$. The studies of Van Mier $[23,24]$ and Van Vliet [25] indicated that the size of the representative volume unit that can reflect the statistical average properties of the material should be at least $7 \sim 8$ times the particle size. Potyondy ad Cundall [7] suggested taking $R_{\max } / R_{\min }=1.66$ without considering the gradation so that the generated model becomes more uniform and conforms to the actual physical properties of the rock. In summary, the minimum radius of the particle unit $R_{\min }$ is $0.3 \mathrm{~mm}$, and the ratio of maximum radius to minimum radius $R_{\max } / R_{\min }$ is 1.66 . The particle unit is randomly generated in accordance with the uniform distribution of the particle size range of $0.3 \sim 0.5 \mathrm{~mm}$ in the designated space. The size information of the model is shown in Table 3, and the established model is shown in Figure 3.

3.2. Parameter Selection. The PBM selected in this study has 12 microparameters. The basic assumptions are as follows: (1) The particle radius ratio is fixed at 1.66. (2) The minimum radius of the particles is $0.3 \mathrm{~mm}$, and the particle units are randomly generated in a uniform distribution within the designated space according to the particle size range of $0.3 \sim 0.5 \mathrm{~mm}$. (3) According to the physical characteristics of dolomite in the Barun open-pit mine, the particle density is equivalent to the rock density of $2950 \mathrm{~kg} / \mathrm{m}^{3}$. (4) The parallel bond strength ratio $K_{\sigma}=\bar{c} / \sigma_{c}$ and the bond tensile strength $\bar{\sigma}_{c}$ are used as the parameters of the particle bond strength to reflect the relationship between the particle bond strength and macro-mechanical properties. 
TABLE 1: The basic mechanical properties of dolomite.

\begin{tabular}{lccc}
\hline Density $\rho$ & $2950 \mathrm{~kg} / \mathrm{m}^{3}$ & The tensile strength TS & $7.84 \mathrm{MPa}$ \\
\hline The uniaxial compressive strength, UCS & $159.65 \mathrm{MPa}$ & The internal friction angle $\theta$ & $53.77^{\circ}$ \\
The elastic modulus, $E$ & $24.16 \mathrm{GPa}$ & The cohesive force $C$ & $21.52 \mathrm{MPa}$ \\
Poisson's ratio, $v$ & 0.238 & & \\
\hline
\end{tabular}

TABle 2: Main macroscopic and microscopic parameters of the PBM.

\begin{tabular}{lccccc}
\hline Macroparameters & \multicolumn{2}{c}{ Particle microparameters } & Bond microparameters \\
\hline UCS & Uniaxial compressive strength $(\mathrm{MPa})$ & $R_{\min }$ & Minimum radius of the particle & $\bar{E}_{c}$ & Bond effective modulus $(\mathrm{GPa})$ \\
$T S$ & Tensile strength $(\mathrm{MPa})$ & $R_{\max } / R_{\min }$ & Radius ratio of the particle & $\bar{k}_{n} / \bar{k}_{s}$ & Bond stiffness ratio (normal-to-shear) \\
$E$ & Elasticity modulus $(\mathrm{GPa})$ & $\rho$ & Density of particle $\left(\mathrm{kg} / \mathrm{m}^{3}\right)$ & $\bar{\sigma}_{c}$ & Bond tensile strength $(\mathrm{MPa})$ \\
$v$ & $\quad$ Poisson's ratio & $E_{c}$ & Effective modulus $(\mathrm{GPa})$ & $\bar{c}$ & Bond cohesion $(\mathrm{MPa})$ \\
$\theta$ & Internal friction angle $/\left(^{\circ}\right)$ & $k_{n} / k_{s}$ & Stiffness ratio (normal-to-shear) & $\phi$ & Bond friction angle $/\left(^{\circ}\right)$ \\
$C$ & Cohesive strength $(\mathrm{MPa})$ & $\mu$ & Friction coefficient & $\lambda$ & Bond radius multiplier \\
\hline
\end{tabular}

TABle 3: Model size information.

\begin{tabular}{|c|c|c|c|c|c|}
\hline \multicolumn{2}{|c|}{ Uniaxial compression test } & \multicolumn{2}{|c|}{ Brazilian split test } & \multicolumn{2}{|c|}{ Triaxial compression test } \\
\hline$L$ & $80 \mathrm{~mm}$ & & & $L$ & $80 \mathrm{~mm}$ \\
\hline$W$ & $40 \mathrm{~mm}$ & $D$ & $40 \mathrm{~mm}$ & $W$ & $40 \mathrm{~mm}$ \\
\hline$R_{\min }$ & $0.3 \mathrm{~mm}$ & $R_{\min }$ & $0.3 \mathrm{~mm}$ & $R_{\min }$ & $0.3 \mathrm{~mm}$ \\
\hline$R_{\max } / R_{\min }$ & 1.66 & $R_{\max } / R_{\min }$ & 1.66 & $R_{\max } / R_{\min }$ & 1.66 \\
\hline$\rho$ & $2950 \mathrm{~kg} / \mathrm{m}^{3}$ & $\rho$ & $2950 \mathrm{~kg} / \mathrm{m}^{3}$ & $\rho$ & $2950 \mathrm{~kg} / \mathrm{m}^{3}$ \\
\hline
\end{tabular}

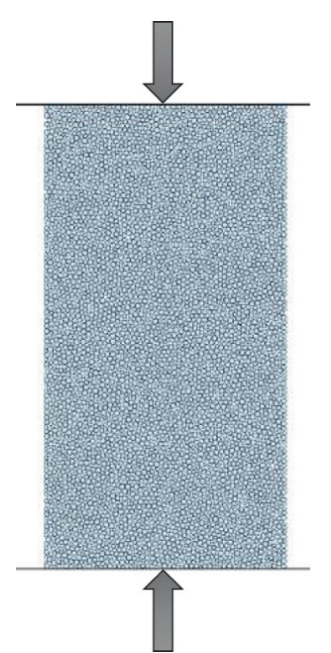

(a)

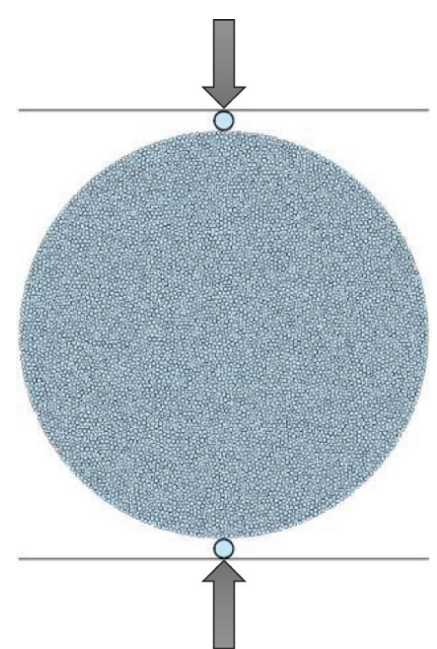

(b)

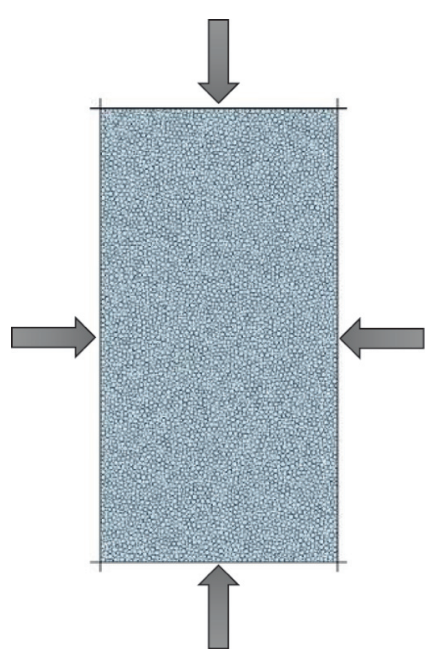

(c)

FIgURE 3: Two-dimensional numerical simulation model. (a) Uniaxial compression test. (b) Brazilian split test. (c) Triaxial compression test.

On the basis of the above assumptions, the microparameters are simplified and adjusted, and nine parameters are selected as the research objects, as shown in Table 4.

3.3. Simulation Test and Results. An orthogonal experiment design uses an orthogonal table to arrange the combination of factors scientifically, thereby reducing the number of experiments and obtaining more reliable experimental results. Through multifactor analysis of variance, the degree of influence of many factors on the test indicators can be evaluated [26]. Therefore, this study uses the orthogonal experimental design method to study the relationship of the macroparameters and microparameters of the PFC2D PBM and quantifies the parameter correspondence relationship to provide a basis for parameter calibration.

According to the number of factors and the level of factors, this study uses the $L_{32}\left(4^{9}\right)$ orthogonal table to design the experiment. There were a total of 32 sets of parameter combinations. On the basis of the parameter combination, numerical tests are performed for uniaxial compression, Brazilian split, and triaxial compression tests (confining 
TABLE 4: Factor level.

\begin{tabular}{lccccc}
\hline Microparameters & \multicolumn{2}{c}{ Factor level } & & Conversion equation \\
\hline$E_{c}$ & 1 & 2 & 3 & 4 & $y=10 x-5$ \\
$E_{c}$ & 5 & 15 & 25 & 35 & $y=10 x-5$ \\
$k_{n} / k_{s}$ & 5 & 15 & 25 & 35 & $y=x$ \\
$\bar{k}_{n} / k_{s}$ & 1 & 2 & 3 & 4 & $y=x$ \\
$\bar{\sigma}_{c}$ & 1 & 2 & 3 & 85 & $y=20 x+5$ \\
$K_{\sigma}$ & 25 & 45 & 1.2 & 1.4 & $y=0.2 x+0.6$ \\
$\mu$ & 0.8 & 1.0 & 0.6 & 0.8 & $y=0.2 x$ \\
$\phi$ & 0.2 & 0.4 & 45 & 60 & $y=15 x$ \\
$\lambda$ & 15 & 30 & 1.0 & 1.2 & $y=0.2 x+0.4$ \\
\hline
\end{tabular}

pressures of 5,10 , and $20 \mathrm{MPa}$, respectively). The specific results of 32 sets of parameter combinations are shown in Table 5.

3.4. Multifactor Analysis of Variance. Multifactor analysis of variance adopts the $F$ test. If the $L_{n}\left(r^{m}\right)$ orthogonal table is used to design the experiment, $y_{1}, y_{2}, \ldots, y_{n}$ are the experimental results, and the basic steps of the analysis of variance are as follows:

(1) Calculate the sum of squares of the total deviations.

$$
\begin{aligned}
S S_{T} & =\sum_{i=1}^{n}\left(y_{i}-\bar{y}\right)^{2} \\
& =\sum_{i=1}^{n} y_{i}^{2}-\frac{1}{n}\left(\sum_{i=1}^{n} y_{i}\right)^{2} .
\end{aligned}
$$

(2) Calculate the sum of squared deviations caused by factor $j$.

$$
S S_{j}=\frac{r}{n}\left(\sum_{i=1}^{r} K_{i}^{2}\right)-\frac{1}{n}\left(\sum_{i=1}^{n} y_{i}\right)^{2},
$$

where $K_{i}$ is the sum of test results corresponding to the $i$-th level of factor $j$.

(3) Calculate $S S_{T}$ and $S S_{j}$ degrees of freedom.

$$
\begin{gathered}
f_{T}=n-1, \\
f_{j}=r-1 .
\end{gathered}
$$

(4) Calculate $F$ statistic.

$$
F_{j}=\frac{S S_{j} / f_{j}}{S S_{T} / f_{T}} .
$$

(5) The original hypothesis of the test is that each factor has no significant effect on the dependent variable. For a given significance level $\alpha$, the critical value $F_{\alpha}$ can be obtained by checking the $F$ distribution table. When the $F$ statistic is greater than $F_{\alpha}$, the influence of the corresponding factor is considered significant; otherwise, it is considered insignificant. SPSS automatically calculates the $F$ value and provides the corresponding concomitant probability value Sig in accordance with the $F$ distribution table. Generally, the significance level of the test is $\alpha=0.05$. If $\operatorname{Sig} \leq \alpha$, then the corresponding factor has a significant effect on the dependent variable; if $\operatorname{Sig}>\alpha$, then the corresponding factor has no significant effect on the dependent variable [20]. To analyze the degree of influence intuitively, the results of the analysis of variance are plotted as a histogram, as shown in Figure 4.

According to the statistical results of the $F$ statistic and the associated probability value Sig of the multifactor analysis of variance, the degree of influence of the test factors on the test indicators varies. The specific analysis is as follows:

(1) The corresponding concomitant probability Sig of bond tensile strength and bond radius multiplier in Figure $4(\mathrm{a})$ is less than 0.05 , which has a significant effect on compressive strength. From the $F$ statistic, the other microparameters have no significant influence on compressive strength.

(2) In Figure 4(b), the tensile strength is mainly affected by the bond tensile strength. Compared with the bond tensile strength, the other microparameters have no significant effect on the tensile strength.

(3) Figure 4(c) shows that the radius multiplier, the effective modulus, and the parallel bonding modulus all have a significant effect on the elastic modulus. The bond effective modulus is the most significant. The associated probability value of the bond tensile strength, the bond strength ratio, and the stiffness ratio is close to 1 , which hardly affects the elastic modulus.

(4) Poisson's ratio in Figure 4(d) is mainly affected by the bond stiffness ratio and the bond effective modulus. Sig of the bond friction angle is close to 1 , and the influence on Poisson's ratio is negligible. The other microparameters have a weak influence on Poisson's ratio.

(5) In Figure 4(e), the main microparameter that affects the size of the internal friction angle is the bond friction angle, and the other microparameters have no significant effect on the internal friction angle. 
TABLE 5: Numerical calculation scheme and results based on the orthogonal experimental design.

\begin{tabular}{|c|c|c|c|c|c|c|c|c|c|c|c|c|c|c|c|}
\hline \multirow{2}{*}{ No. } & \multicolumn{9}{|c|}{ Microparameters } & \multicolumn{6}{|c|}{ Macroparameters } \\
\hline & $E_{c}(\mathrm{GPa})$ & $E_{c}(\mathrm{GPa})$ & $k_{n} / k_{s}$ & $\bar{k}_{n} / \bar{k}_{s}$ & $\bar{\sigma}_{c}(\mathrm{MPa})$ & $K_{\sigma}$ & $\mu$ & $\phi\left({ }^{\circ}\right)$ & $\lambda$ & UCS (MPa) & TS (MPa) & $E(\mathrm{GPa})$ & $v$ & $\theta\left(^{\circ}\right)$ & $C(\mathrm{MPa})$ \\
\hline 1 & 5 & 5 & 1 & 1 & 25 & 0.8 & 0.2 & 15 & 0.6 & 25.53 & 2.11 & 7.01 & 0.21 & 17.91 & 9.46 \\
\hline 2 & 5 & 5 & 4 & 2 & 65 & 1.4 & 0.6 & 60 & 0.8 & 122.73 & 11.18 & 7.46 & 0.31 & 42.76 & 27.77 \\
\hline 3 & 5 & 15 & 3 & 4 & 85 & 1.0 & 0.2 & 60 & 1.2 & 188.02 & 14.90 & 19.03 & 0.33 & 44.02 & 42.41 \\
\hline 4 & 5 & 15 & 2 & 3 & 45 & 1.2 & 0.6 & 15 & 1.0 & 114.13 & 9.29 & 17.70 & 0.26 & 32.23 & 33.36 \\
\hline 5 & 5 & 25 & 1 & 3 & 65 & 1.2 & 0.4 & 45 & 1.2 & 196.70 & 9.93 & 30.90 & 0.24 & 45.15 & 42.17 \\
\hline 6 & 5 & 25 & 4 & 4 & 25 & 1.0 & 0.8 & 30 & 1.0 & 55.72 & 3.89 & 25.04 & 0.28 & 34.63 & 15.75 \\
\hline 7 & 5 & 35 & 3 & 2 & 45 & 1.4 & 0.4 & 30 & 0.6 & 69.24 & 3.03 & 24.41 & 0.19 & 31.00 & 20.77 \\
\hline 8 & 5 & 35 & 2 & 1 & 85 & 0.8 & 0.8 & 45 & 0.8 & 126.69 & 10.46 & 36.08 & 0.06 & 35.16 & 33.50 \\
\hline 9 & 15 & 5 & 4 & 1 & 45 & 1.0 & 0.4 & 45 & 1.0 & 107.42 & 7.65 & 15.62 & 0.30 & 31.25 & 31.09 \\
\hline 10 & 15 & 5 & 1 & 2 & 85 & 1.2 & 0.8 & 30 & 1.2 & 323.64 & 27.26 & 17.20 & 0.29 & 42.80 & 71.57 \\
\hline 11 & 15 & 15 & 2 & 4 & 65 & 0.8 & 0.4 & 30 & 0.8 & 111.07 & 7.78 & 19.77 & 0.42 & 35.34 & 29.44 \\
\hline 12 & 15 & 15 & 3 & 3 & 25 & 1.4 & 0.8 & 45 & 0.6 & 39.13 & 2.45 & 17.68 & 0.35 & 40.37 & 9.75 \\
\hline 13 & 15 & 25 & 4 & 3 & 85 & 1.4 & 0.2 & 15 & 0.8 & 154.46 & 14.54 & 27.84 & 0.35 & 39.56 & 37.98 \\
\hline 14 & 15 & 25 & 1 & 4 & 45 & 0.8 & 0.6 & 60 & 0.6 & 42.76 & 2.49 & 23.53 & 0.34 & 44.18 & 9.65 \\
\hline 15 & 15 & 35 & 2 & 2 & 25 & 1.2 & 0.2 & 60 & 1.0 & 59.93 & 3.29 & 45.56 & 0.21 & 43.68 & 13.41 \\
\hline 16 & 15 & 35 & 3 & 1 & 65 & 1.0 & 0.6 & 15 & 1.2 & 155.80 & 12.66 & 61.76 & 0.07 & 24.63 & 51.32 \\
\hline 17 & 25 & 5 & 2 & 3 & 85 & 1.0 & 0.4 & 60 & 0.6 & 86.93 & 7.64 & 13.24 & 0.82 & 38.39 & 21.65 \\
\hline 18 & 25 & 5 & 3 & 4 & 45 & 1.2 & 0.8 & 15 & 0.8 & 86.94 & 6.49 & 15.71 & 0.65 & 37.52 & 22.35 \\
\hline 19 & 25 & 15 & 4 & 2 & 25 & 0.8 & 0.4 & 15 & 1.2 & 64.75 & 2.69 & 33.84 & 0.30 & 28.24 & 20.06 \\
\hline 20 & 25 & 15 & 1 & 1 & 65 & 1.4 & 0.8 & 60 & 1.0 & 188.76 & 12.26 & 38.07 & 0.13 & 45.70 & 41.49 \\
\hline 21 & 25 & 25 & 2 & 1 & 45 & 1.4 & 0.2 & 30 & 1.2 & 147.01 & 6.56 & 54.17 & 0.13 & 34.35 & 40.95 \\
\hline 22 & 25 & 25 & 3 & 2 & 85 & 0.8 & 0.6 & 45 & 1.0 & 187.94 & 8.89 & 42.51 & 0.27 & 40.93 & 43.71 \\
\hline 23 & 25 & 35 & 4 & 4 & 65 & 1.2 & 0.2 & 45 & 0.6 & 83.38 & 7.36 & 31.92 & 0.45 & 38.33 & 20.74 \\
\hline 24 & 25 & 35 & 1 & 3 & 25 & 1.0 & 0.6 & 30 & 0.8 & 50.79 & 1.67 & 43.10 & 0.26 & 38.43 & 12.67 \\
\hline 25 & 35 & 5 & 3 & 3 & 65 & 0.8 & 0.2 & 30 & 1.0 & 135.32 & 9.93 & 18.20 & 0.84 & 23.43 & 44.96 \\
\hline 26 & 35 & 5 & 2 & 4 & 25 & 1.4 & 0.6 & 45 & 1.2 & 71.92 & 5.06 & 22.57 & 0.62 & 38.26 & 18.20 \\
\hline 27 & 35 & 15 & 1 & 2 & 45 & 1.0 & 0.2 & 45 & 0.8 & 85.68 & 5.80 & 30.46 & 0.48 & 29.83 & 25.06 \\
\hline 28 & 35 & 15 & 4 & 1 & 85 & 1.2 & 0.6 & 30 & 0.6 & 146.48 & 9.36 & 32.29 & 0.34 & 30.19 & 42.46 \\
\hline 29 & 35 & 25 & 3 & 1 & 25 & 1.2 & 0.4 & 60 & 0.8 & 45.67 & 2.89 & 46.31 & 0.17 & 38.63 & 11.79 \\
\hline 30 & 35 & 25 & 2 & 2 & 65 & 1.0 & 0.8 & 15 & 0.6 & 101.88 & 6.11 & 37.18 & 0.32 & 33.04 & 28.58 \\
\hline 31 & 35 & 35 & 1 & 4 & 85 & 1.4 & 0.4 & 15 & 1.0 & 216.12 & 10.24 & 52.94 & 0.39 & 44.00 & 48.27 \\
\hline 32 & 35 & 35 & 4 & 3 & 45 & 0.8 & 0.8 & 60 & 1.2 & 106.53 & 5.67 & 62.55 & 0.29 & 47.14 & 21.89 \\
\hline
\end{tabular}

(6) Figure 4(f) shows that the bond cohesion is affected by various microparameters, including bond tensile strength, bond radius multiplier, bond friction angle, and bond stiffness ratio. The influence of bond tensile strength and the bond radius multiplier is the most evident.

Multifactor analysis of variance selects the factors that have a significant effect on the test indicators from numerous microparameters. Through regression analysis of significant influencing factors and test indicators, the corresponding linear and nonlinear relationships between macroparameters and microparameters are obtained.

$$
\begin{aligned}
\mathrm{UCS} & =2.12 \bar{\sigma}_{c}+141.15 \lambda-127.85, \\
T S & =0.17 \bar{\sigma}_{c}-1.35, \\
E & =0.56 E c+1.00 \bar{E}_{c}+23.32 \lambda-21.90, \\
v & =-0.08 \ln \bar{E}_{c}+0.11 \ln \bar{k}_{n} / \bar{k}_{s}+0.31, \\
\theta & =0.004 \phi^{2}-0.09 \phi+32.52,
\end{aligned}
$$

$$
C=-11.76 \ln \bar{k}_{n} / \bar{k}_{s}+0.48 \bar{\sigma}_{c}-0.20 \phi+31.75 \lambda-14.24 \text {. }
$$

3.5. Calibration of Microparameters. The results of the analysis of variance show that the number and types of microparameters that have a significant effect on different test indicators vary. The parallel bond strength ratio, friction coefficient, and stiffness ratio have the associated probability value Sig greater than 0.05 , indicating that it has no significant effect on the macroparameters. These factors are secondary factors that affect the macroparameters. Therefore, the following assumptions are made:

(1) By observing the failure mode of specimens in the uniaxial compression test, it can be found that the final failure mode of the simulated specimen is different for the different bond strength ratios. In order to study the relationship between them, 4 sets of uniaxial compression are performed by the controlled variable method. Ensure that the remaining parameters remain unchanged (the same as the 16th set of the orthogonal test), and the bond strength 


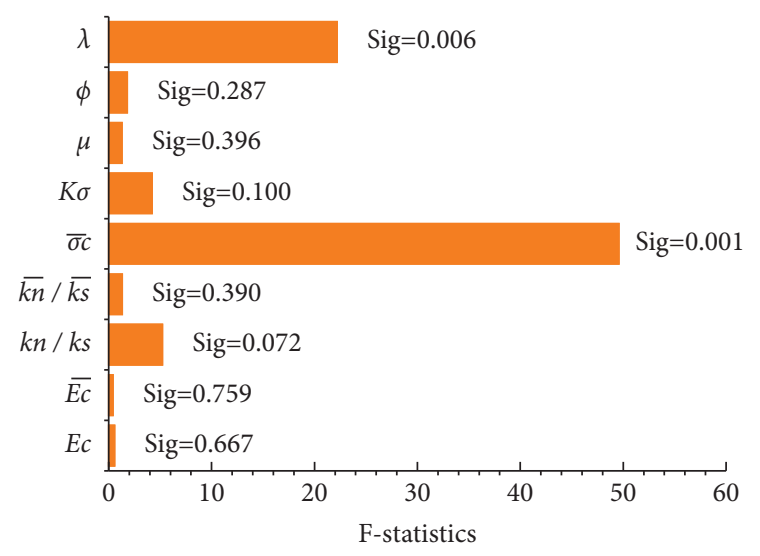

(a)

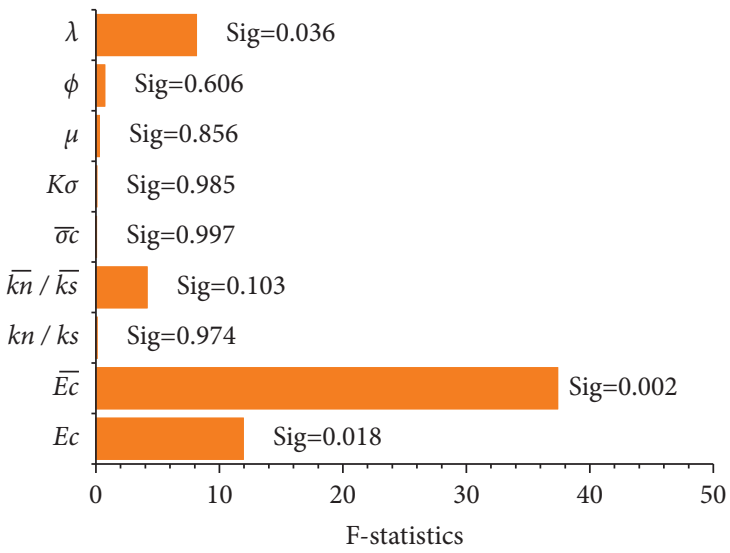

(c)

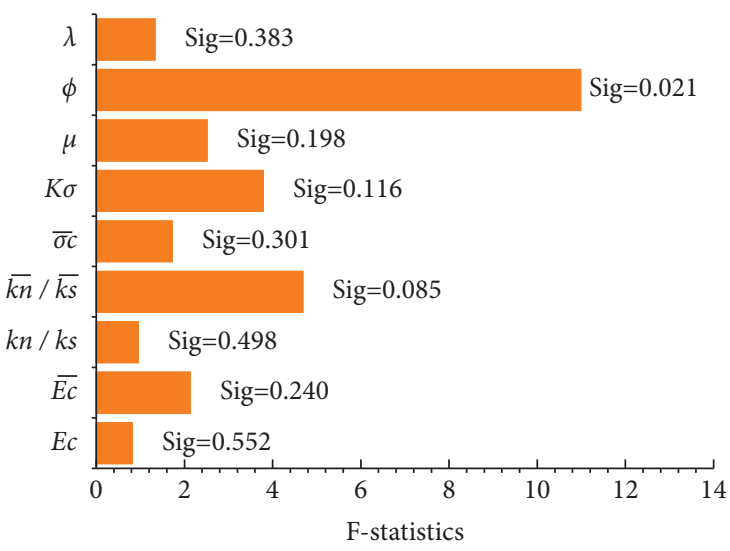

(e)

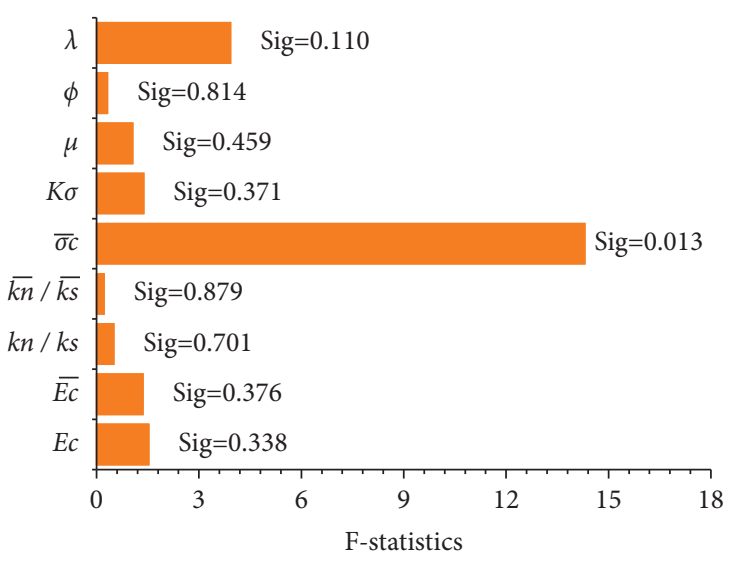

(b)

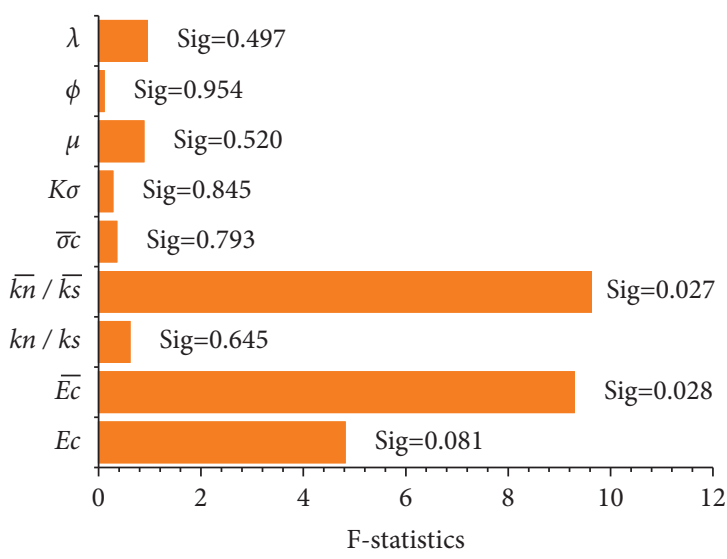

(d)

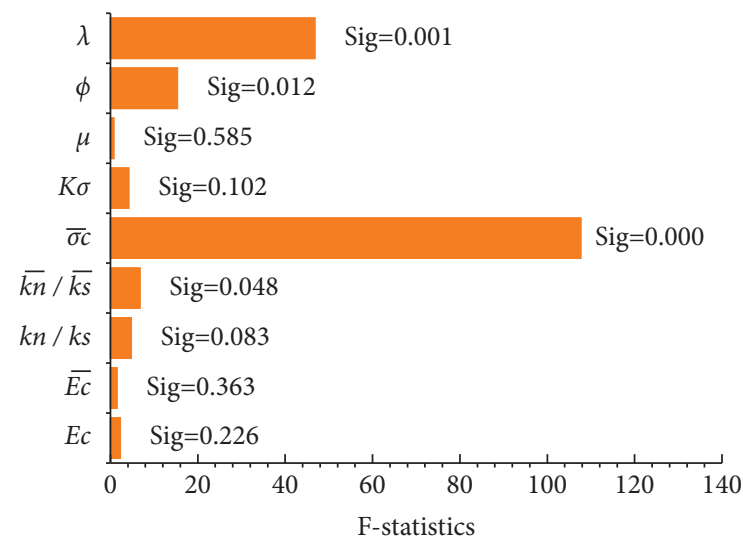

(f)

FIgURE 4: $F$ statistics of multifactor analysis of variance. (a) Uniaxial compressive strength. (b) Tensile strength. (c) Elasticity modulus. (d) Poisson's ratio. (e) Internal friction angle. (f) Cohesive strength.

ratios are, respectively, $0.8,1.0,1.2$, and 1.4. The final failure modes of specimens with different bond strength ratios are shown in Figure 5. It can be seen that when the value of bond tensile strength remains unchanged, the smaller the bond strength ratio is, the easier it is to produce shear failure and the more obvious the failure surface is; the larger the bond strength ratio is, the easier it is to produce conjugation failure and the less obvious the failure surface is. Because the bond strength of the material is affected by the mutual influence of bond tensile strength and bond cohesion, the failure form of the particle bonding point mainly depends on who reaches the failure point first. Under the condition that the value of bond tensile strength remains unchanged, the smaller the bond cohesion is, the more likely the particles are to produce tangential failure, which is beneficial to the formation of the 

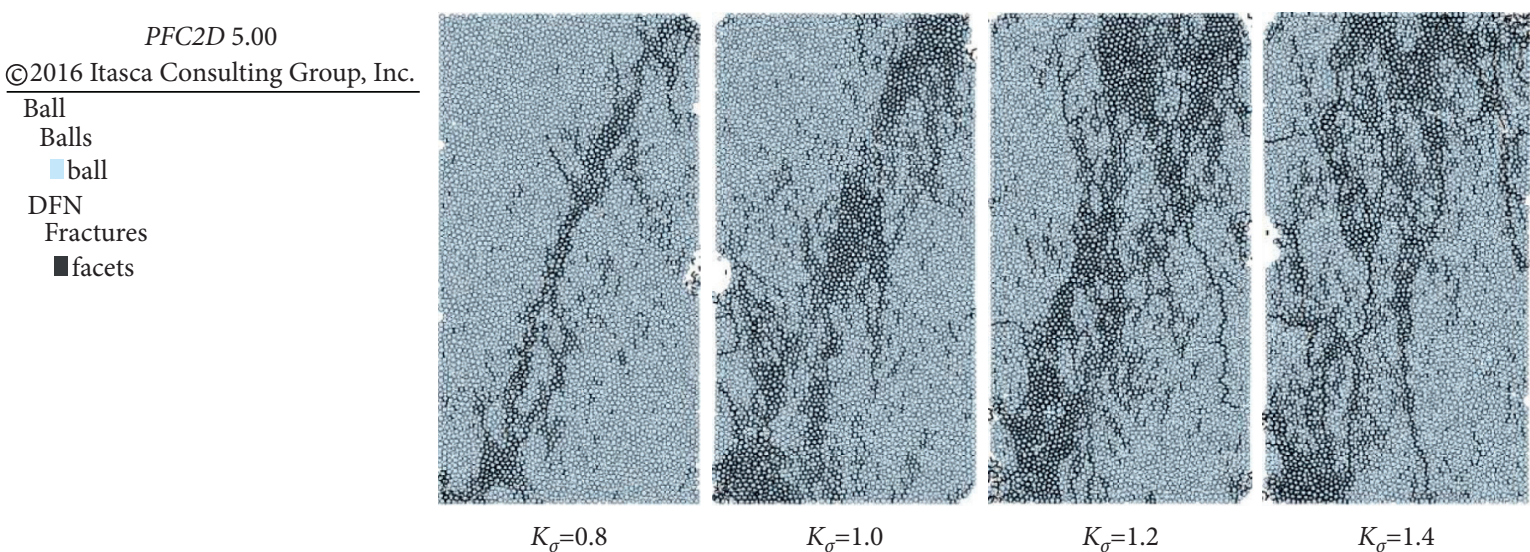

FIGURE 5: Rock failure pattern at different bond strength ratios (tangential/normal) in uniaxial compression test.

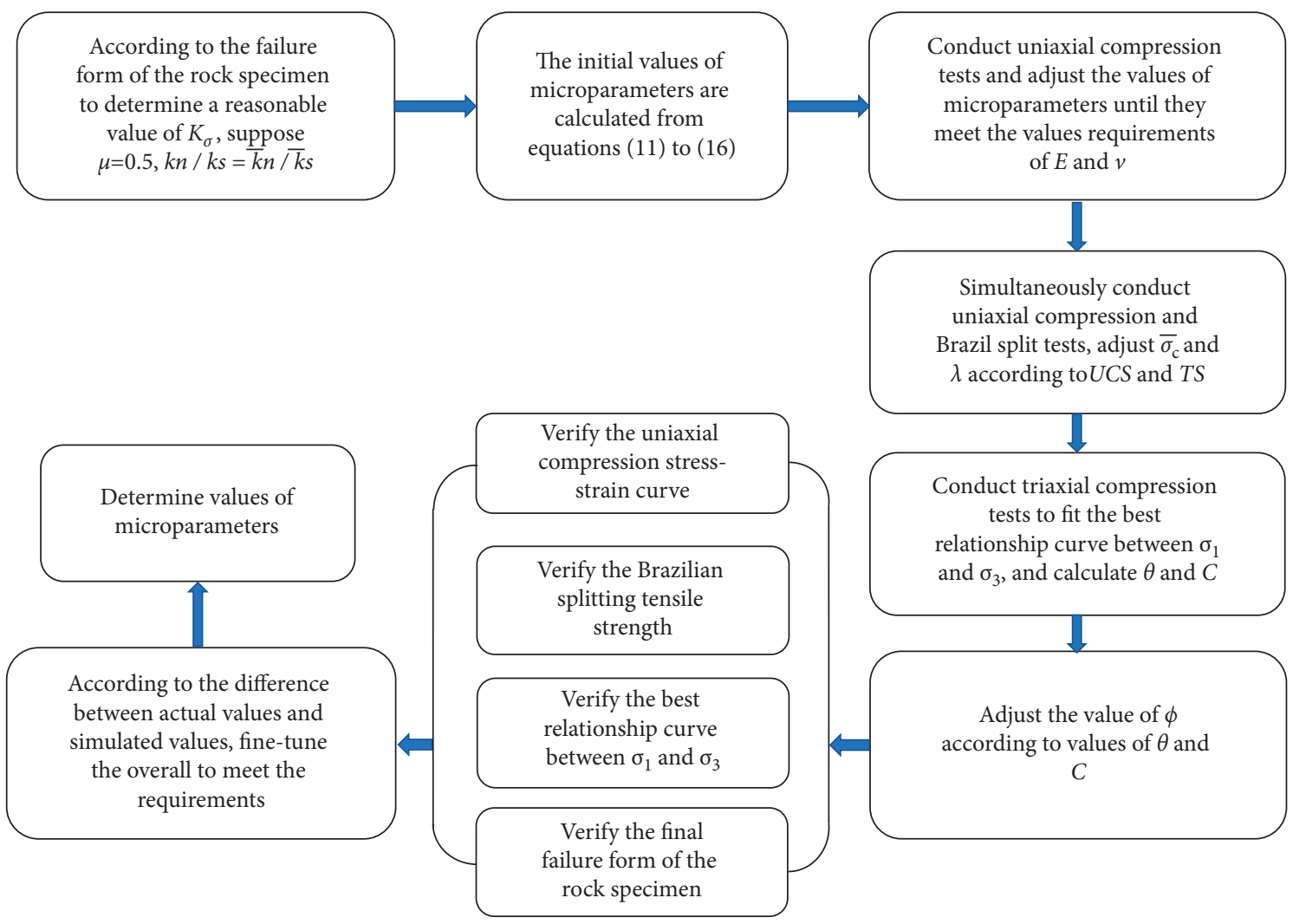

FIGURE 6: Flowchart of the calibration of microparameters.

shear failure surface. Therefore, a reasonable bond strength ratio can be selected in accordance with the actual failure form of the test block.

(2) The friction coefficient has no significant effect on the macroparameters. In the particle discrete element model, the particles start to slip after the bond is broken. The friction coefficient between particles only affects the mechanical behavior of the material after reaching the stress peak. The mechanical response after the peak is difficult to describe quantitatively. Therefore, in accordance with the suggestion of Potyonndy and Cundall [7], the general empirical value of 0.5 can be adopted for the friction coefficient of rock-like materials.

(3) In the PBM, Cundalll [27] set $k_{n} / k_{s}=\bar{k}_{n} / \bar{k}_{s}$ to simulate the mechanical behavior of rocks. The results show that this setting can meet the characteristics of rock materials well.

On the basis of the above assumptions and in accordance with the macroparameter and microparameter equations of formulae (11) (16), the initial microparameters are obtained. Afterward, the principle of "less first, then more, easy first, and then difficult" is adopted to determine the parameter adjustment sequence. The specific process is shown in Figure 6. 
TABle 6: Process of dolomite microparameter calibration.

\begin{tabular}{lcccccccccccccccc}
\hline \multicolumn{1}{c}{ Step } & \multicolumn{10}{c}{ Microparameters } \\
& $E_{c}(\mathrm{GPa})$ & $\bar{E}_{c}(\mathrm{GPa})$ & $k_{n} / k_{s}$ & $\bar{k}_{n} / \bar{k}_{s}$ & $\bar{\sigma}_{c}(\mathrm{MPa})$ & $K_{\sigma}$ & $\mu$ & $\phi\left(^{\circ}\right)$ & $\lambda$ & $\mathrm{UCS}(\mathrm{MPa})$ & $T S(\mathrm{MPa})$ & $E(\mathrm{GPa})$ & $v$ & $\theta\left({ }^{\circ}\right)$ & $C(\mathrm{MPa})$ \\
\hline 1 & 15.9 & 9.2 & 2.6 & 2.6 & 54.1 & 0.8 & 0.5 & 85.0 & 1.2 & 111.03 & 5.52 & 20.68 & 0.301 & 57.85 & 18.91 \\
2 & 17.5 & 10.5 & 2.3 & 2.3 & 54.1 & 0.8 & 0.5 & 85.0 & 1.2 & 109.54 & - & 23.74 & 0.269 & - & - \\
3 & 17.5 & 10.5 & 2.3 & 2.3 & 70.0 & 0.8 & 0.5 & 85.0 & 1.4 & 155.13 & 7.07 & 23.61 & 0.273 & - & - & - \\
4 & 17.5 & 10.5 & 2.3 & 2.3 & 80.0 & 0.8 & 0.5 & 85.0 & 1.4 & 168.17 & 7.89 & 23.53 & 0.270 & 58.11 & 23.01 \\
5 & 17.5 & 10.5 & 2.2 & 2.2 & 80.0 & 0.8 & 0.5 & 85.0 & 1.3 & 147.25 & 7.01 & 23.77 & 0.261 & 58.27 & 23.65 \\
6 & 17.5 & 10.5 & 2.1 & 2.1 & 85.0 & 0.8 & 0.5 & 85.0 & 1.3 & 151.68 & 7.24 & 24.91 & 0.258 & 57.51 & 23.04 \\
\hline
\end{tabular}

TABLE 7: Actual and simulated values of the macroparameters of dolomite.

\begin{tabular}{lcccccc}
\hline Macroparameters & UCS $(\mathrm{MPa})$ & TS $(\mathrm{MPa})$ & $E(\mathrm{GPa})$ & $v$ & $\theta\left(^{\circ}\right)$ & $C(\mathrm{MPa})$ \\
\hline Values of the actual & 159.65 & 7.84 & 24.16 & 0.238 & 53.77 \\
Values of simulation & 151.68 & 7.24 & 24.91 & 0.258 & 57.51 & 21.52 \\
Error $(\%)$ & 4.99 & 7.65 & 3.10 & 8.40 & 6.96 & 7.06 \\
\hline
\end{tabular}

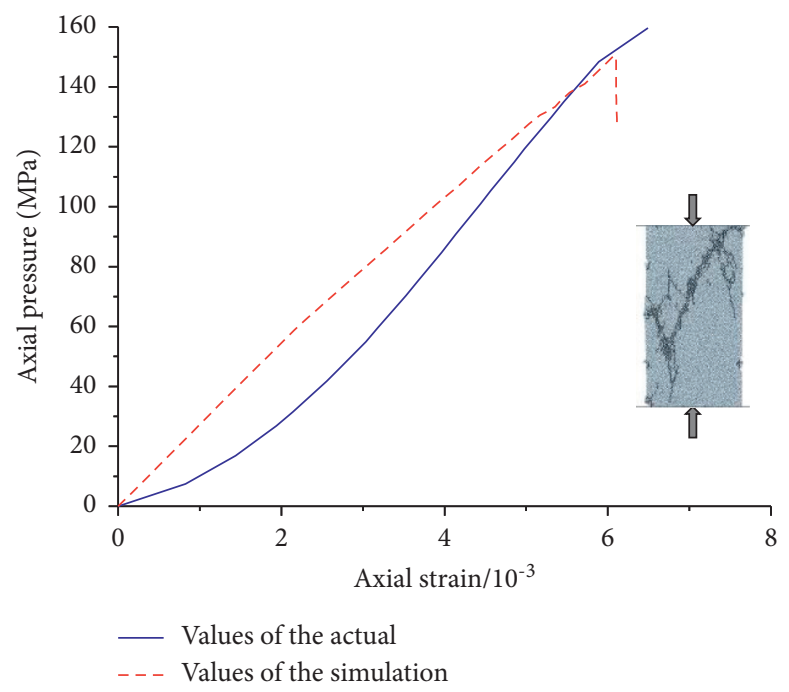

FIgURE 7: Uniaxial compression stress-strain curve of the actual and numerical simulation tests.

\section{Instance Verification}

The above method is used to calibrate the microparameters of dolomite in the Barun open-pit mine. Table 6 reproduces the microparameter calibration process, which has been adjusted six times in total. Equations (11) (16) can be inversely calculated to obtain the initial microparameters of step 1. At this time, a certain gap remains between the corresponding macroparameters and the target value because of numerous microparameters; in addition, only regression microparameters that have a significant effect on macroparameters are considered, whereas parameters that have a nonsignificant effect may have a certain effect on the test results. Steps $2 \sim 6$ refer to the calibration process in Figure 6 to adjust the microparameters appropriately. The actual value of the test and the simulation value are summarized in Table 7 . The error between the simulation result and the target value is within $8.5 \%$. Therefore, the calibration result meets the requirements.

The uniaxial compression stress-strain curve and Brazilian split stress-strain curve are obtained by numerical simulation and actual physical tests (Figures 7 and 8 ). The curve trends of the numerical simulation and actual physical tests are the same, but certain differences can be observed. The microcracks in the dolomite specimens are closed under pressure, and the stress-strain curve has an evident compaction stage. The numerical simulation uses a constant servo pressure when generating particles to make the resulting particle assembly contact dense and uniform. Thus, the prepeak stage of the stress-strain relationship is approximately a straight line.

Figure 9 shows the comparison of the relationship curve between the limit principal stresses of the numerical test and the actual test. When the confining pressure is small, the ultimate axial stress predicted by the numerical model is closer to the test result. As the confining pressure increases, the difference between them also gradually increases. The reason may be that PFC is based on the discrete element method, which treats rock-like materials as an aggregate of spherical particles, whereas the actual rock and mineral particle shapes are irregular polyhedrons. The difference in shape may cause differences in the occlusal function of the particles, and the increase in confining pressure amplifies this difference. 


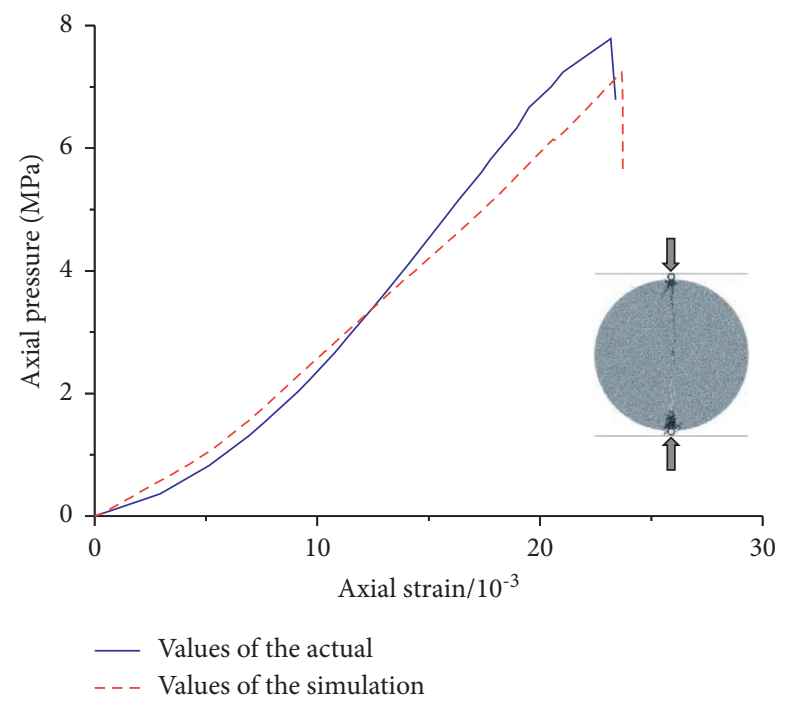

FIGURE 8: Brazilian split stress-strain curve of the actual and numerical simulation tests.

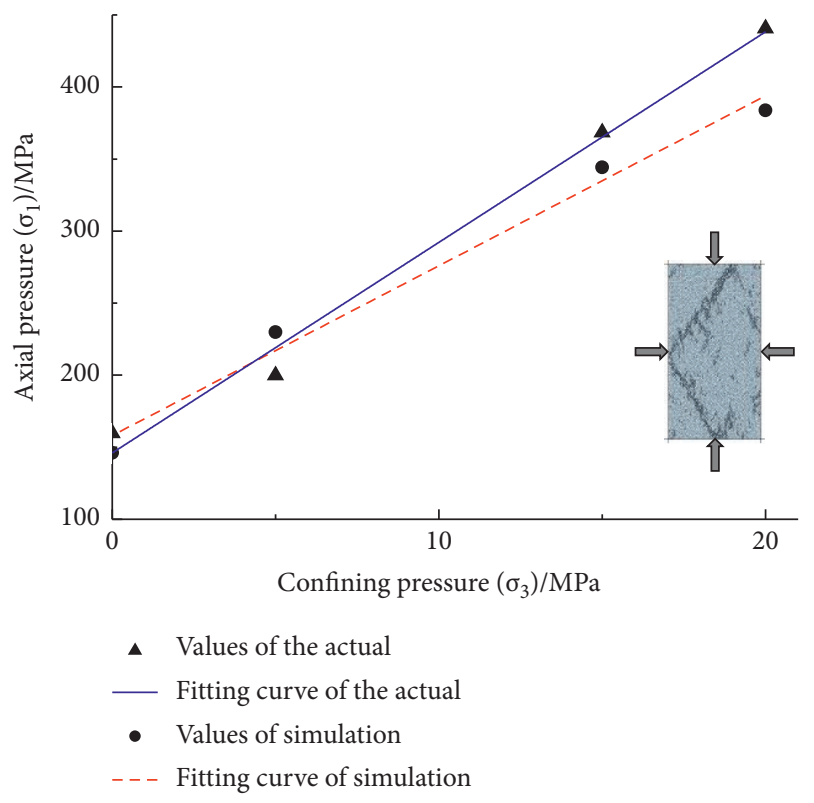

FIGURE 9: Triaxial compression ultimate principal stress $\left(\sigma_{1} \sim \sigma_{3}\right)$ relationship curve.

\section{Conclusion}

Multifactor analysis of variance was performed on the results of the orthogonal experiment, and the degree of influence of each microparameter on the macroparameters was accurately estimated. In accordance with the results of the analysis of variance and by comprehensively considering the influence of multiple microparameters on the test indicators, a multivariate functional relationship between the significant influencing factors and the test indicators was established.

(1) The uniaxial compressive strength of rock is mainly affected by the bond tensile strength and the bond radius multiplier.
(2) The rock tensile strength is mainly affected by the bond tensile strength, and the two parameters have a linear relationship.

(3) microparameters that have a significant effect on the elasticity modulus of the rock, the degree of influence from high to low is bond effective modulus, effective modulus, and bond radius multiplier.

(4) Rock Poisson's ratio is mainly affected by the bond stiffness ratio and the bond effective modulus.

(5) The macroscopic variable internal friction angle of rock is mainly affected by the bond friction angle, and the internal friction angle varies with the increase in bond friction angle.

(6) The types of microparameters that have a significant effect on cohesive strength and its degree of influence in descending order are as follows: bond tensile strength, bond radius multiplier, bond friction angle, and bond stiffness ratio.

In accordance with the relationship between the macroparameters and microparameters, the calibration order of the microparameters was determined, and a specific calibration method was proposed. This method was used to calibrate the mechanical parameters of dolomite in the Barun open-pit mine. The simulation test results were consistent with the actual test results, thus providing a basis for a subsequent numerical simulation study on the mechanical properties of dolomite.

\section{Data Availability}

The data included in this study are available from the corresponding author upon request.

\section{Conflicts of Interest}

The authors declare that there are no conflicts of interest regarding the publication of this paper. 


\section{References}

[1] P. A. Cundall and O. D. L. Strack, "A discrete numerical model for granular assemblies," Géotechnique, vol. 29, no. 1, 1979.

[2] H. C. Zhu, "PFC and application case of caving study," Chinese Journal of Rock Mechanics and Engineering, vol. 9, pp. 1927-1932, 2006.

[3] S. X. Deng, Y. L. Zheng, and L. P. Feng, "Application of design of experiments in microparameters calibration for hard rocks of PFC3D model," Chinese Journal of Geotechnical Engineering, vol. 41, no. 4, pp. 655-664, 2019.

[4] M. J. Jiang, W. Fang, and J. Sima, "Calibration of microparameters of parallel bonded model for rocks," Journal of Shandong University Engineering Science Edition, vol. 45, no. 4, pp. 50-56, 2015.

[5] X. R. Liu, Y. Fu, and Y. R. Zheng, "Relation between microparameters of particle flow code and fracture toughness of rock," Chinese Journal of Rock Mechanics and Engineering, vol. 30, no. 10, pp. 2084-2089, 2011.

[6] Y. Cong, Z. Q. Wang, and Y. R. Zheng, "Experimental study on microparameters of brittle materials based on particle flow theory," Chinese Journal of Geotechnical Engineering, vol. 37, no. 6, pp. 1031-1040, 2015.

[7] D. O. Potyondy and P. A. Cundall, "A bonded-particle model for rock," International Journal of Rock Mechanics and Mining Sciences, vol. 41, no. 8, 2004.

[8] J. M. Xu, Z. L. Xie, and H. T. Jia, "Simulation of micromechanical properties of limestone using particle flow code," Rock and Soil Mechanics, vol. 31, no. 2, pp. 390-395, 2010.

[9] A. Nardin and B. A. Schrefler, "Modeling of cutting tool soil interaction part III: macromechanical model and upscaling," Computer Mechanics, vol. 36, no. 5, pp. 343-359, 2005.

[10] B. D. Yang, Y. Jiao, and S. T. Lei, "A study on the effects of microparameters on macroproperties for specimens created by bonded particles, Engineering Computations," International Journal for Computer-Aided Engineering and Software, vol. 23, no. 6, 2006.

[11] H. Y. Huang, Discrete Element Modeling of Tool Rock Interaction, University of Minnesota, Minneapolis, MN, USA, 1999.

[12] G. Y. Zhao, B. Dai, and C. Ma, "Study of effects of microparameters on macroparameters for parallel bonded mode," ChineseJournalof Rock Mechanics and Engineering, vol. 31, no. 7, pp. 3171-3178, 2012.

[13] J. Yan, Y. W. Zeng, and R. Gao, "Relationship between macroparameters and microparameters in particle flow model of unbonded material," Journal of Yangtze River Scientific Research Institute, vol. 29, no. 5, pp. 45-50, 2012.

[14] Y. D. Chen, Y. Yu, and Y. X. She, "Method for determining microparameters of sand in three-dimensionalparticle flow code numerical modeling," Chinese Journal of Geotechnical Engineering, vol. 35, no. 2, pp. 88-93, 2013.

[15] Y. Zhou, S. C. Wu, and J. J. Jiao, "Research on mesomechanical parameters of rock and soil mass based on BP neural network II," Rock and Soil Mechanics, vol. 32, no. 12, pp. 3821-3826, 2011.

[16] L. X. Niu and Y. Y. Xin, "Analysis on relationship between macroparameters and microparameters in particle flow modle based on orthogonal design: case of rock uniaxial compression numerical test," Yangtze River, vol. 46, no. 16, pp. 53-57, 2015.

[17] P. Y. Chen, Y. Kong, and H. M. Yu, "Research on the calibration method of microparameters of a uniaxial compression pfc2d model for rock," Chinese Journal of Underground Space and Engineering, vol. 14, no. 5, pp. 1240-1249, 2018.

[18] B. Q. Hao, S. C. Zhang, and C. L. Wang, "Research on Calibration Method of Microparameters of PFC2D Model for Rock," Coal Science and Technology, pp. 1-11, New York, NY, USA, 2021.

[19] Z. M. Yin, X. G. Wang, and D. S. Wang, "Analysis and application of stress distribution in 24-m high bench loosening blasting with axially uncoupled charge structure in Barun Open-pit Mine," IOP Conference Series: Earth and Environmental Science, vol. 804, no. 2, 2021.

[20] C. Shi, Q. Zhang, and S. N. Wang, Numerical Simulation Technology and Application with Particle Flow code(PFC5.0), China Architecture \& Building Press, Beijing, China, 2018.

[21] E. D. Abi, Y. R. Zheng, and X. T. Feng, "Relationship between particle micro and macro mechanical parameters of parallel bond model," Rock and Soil Mechanics, vol. 39, no. 4, pp. 1289-1301, 2018.

[22] X. T. Yin, Y. N. Zheng, and S. K. Ma, "Study of inner scale ratio of rock and soil material based on numerical tests of particle flow code," Rock and Soil Mechanics, vol. 32, no. 4, pp. 1211-1215, 2011.

[23] J. G. M. VanMier, "Microstructural effects on fracture scaling in concrete, Rock and Ice," in Proceedings of the IUTAM Symposium on ScalingLaws in Ice Mechanics and Ice Dynamics, Alaska, AK, USA, June 2001.

[24] J. G. M. VanMier, Fracture Processes of concrete, CRC Press, Boca Raton, FL, USA, 1996.

[25] M. R. A. V. Vliet and, Size Effect in Tensile Fracture of concrete and Rock, Delft University of Technology, Delft, Netherlands, 2000.

[26] W. T. Zhang, SPSS Statistical Analysis Basic Tutorial, Higher Education Press, Beijing, China, 2017.

[27] P. A. Cundall, "Formulation of a three-dimensional distinct element modela part I. Ascheme to detect and represent contacts in a system composed of many polyhedralblocks," International Journal of Rock Mechanics and Mining Science \& Geomechanics Abstracts, vol. 25, no. 3, 1988. 